\section{Breakdown of Chemicals}

\author{
from a Correspondent
}

Pollution dangers can be reduced and persistence controlled as long as the research effort into the breakdown of man-made chemicals in the environment can keep pace with their usage. There is urgent need for more work in this field with emphasis on greater cooperation between microbiologists, biochemists and chemists. These were the messages to come out of the symposium on microbiological aspects of the breakdown of chemicals in the environment arranged by the Microbiology Group of the Society of Chemical Industry in London on February 3.

The morning session began with a contribution by Dr H. A. Painter (Water Pollution Research Laboratory, Stevenage), who pointed out that the growth of nitrifying microbes requires oxygen and is very susceptible to inhibition by chelating agents, metals and chlorate. $\mathrm{He}$ emphasized the need to adopt acceptable and reproducible techniques for obtaining, storing and analysing water samples. Miss S. Beastall and Professor D. E. Hughes (University College, Cardiff) dealt with the microbial breakdown of crude oils. Work at Cardiff had shown that hydrocarbonoxidizing microbes may comprise 2.5 to 9 per cent of the microbial population of the Bristol Channel. The main factors limiting microbial breakdown of oils are availability of oxygen and mineral nutrients. Methods of combating oil spills are being sought and promising developments include use of biodegradable non-toxic emulsifiers and sinking agents.

Dr J. H. Walsh (University of Manchester Institute of Science and Technology) said that most plastics are not very susceptible to biodegradation and microbial growth often associated with degraded plastic is likely to be on plasticizers or other additives. It was possible, however, that polyurethanes, many of which are degraded by fungi, may be chemically designed so as to exhibit a predictable susceptibility and hence persistence. Mutation or "training" processes enabling microbes to attack plastic polymers seemed unlikely.

In the afternoon session Dr A. J. Willets (University of Exeter) described the degradation of alkyl benzene sulphonate (ABS) surfactants. Many common microorganisms degrade these compounds and use similar metabolic routes, the first and key step being desulphonation followed by oxidation of the alkyl chain and then aromatic ring cleavage. Dr I. Hill (ICI Ltd, Jealott's Hill) pleaded for an examination of pollution of the soil by pesticides. Having described reactions often involved in

pesticide degradation he suggested that chemists should look to the design of biodegradable insecticides. Dr S. J. L. Wright (University of Bath) described the microbial metabolism of phenylamide herbicides. Aniline compounds which are formed as metabolites of such herbicides may in turn either be metabolized or, as in the case of some chloroanilines, condense tc form azobenzenes or combine with persistent humic materials. Appreciation of microbiological factors involved in herbicide degradation in certain cases suggested possibilities for both extending and reducing persistence in soil. Several speakers pointed to deficiencies in the "pureculture" experimental approach to biodegradation problems and suggested that it might be more realistic to use mixed cultures and co-metabolic techniques.

Dr K. Mellanby (Monks Wood Experimental Station) summed up by saying that as long as sufficient effort is directed to understanding the fate of man-made chemicals in the environment, pollution dangers will be minimized and people may continue to enjoy the benefits of their use.

\section{The Stute of Rhodopsin}

Two articles in next Wednesday's Nature New Biology (March 15) present new data about the physical state of rhodopsin in the retinal rod membrane. Rhodopsin is a protein, probably of molecular weight 40,000 , containing the visual chromophore 11-cis retinal. It is known that rods are strongly dichroic, the long axis of the retinal being aligned essentially perpendicularly to the rod axis. In the perpendicular plane, by contrast, there is no measurable dichroism, indicating random rotational orientation. More than ten years ago, Hagins and Jennings found that partial bleaching with polarized light did not result in the generation of dichroism. This was surprising, because the molecules oriented with the chromophore in the direction of polarization should be bleached, leaving those more nearly perpendicular to this direction intact.

P. K. Brown has now confirmed this finding, and he has further shown that if the retinas are first treated with the cross-linking agent, glutaraldehyde, a quite different result is obtained: a high degree of dichroism appears, which increases progressively with the extent of bleaching. In control experiments with the monofunctional formaldehyde no dichroism was generated. These results discriminate between the two obvious explanations of the absence of dichroism after polarized bleaching of untreated retinas, in that they exclude a mechanism involving transfer of excitation energy between chromophores

\section{RIBOSOMES}

\section{Contemplating Cores}

from our Molecular Biology Correspondent

Whatever other marks they may leave on the field, ribosomologists seem rarely to have neglected to contribute their very own nomenclature for ribosomal proteins and factors. This invests the literature on the subject with that curiously impenetrable quality which repels most outsiders. Given patience, however, some new results of no small interest can be quarried from recent work of a number of groups.

Allet (Europ. J. Biochem., 24, 421; 1972) shows, by analysis of the proteins shed and retained by $50 \mathrm{~S}$ subunits of Escherichia coli ribosomes on treatment with high salt concentrations, that the division into "split" and "core" proteins is in a sense arbitrary. That is to say, there is a spread of effective binding constants, modulated by the conditions. Using lower than customary magnesium concentrations (in the region of millimolar), Allet finds that lithium and caesium chloride produce similar before the photochemical bleaching event. Such transfer would be unaffected by cross-linking. It may be inferred instead that the rhodopsin molecules are free to rotate about an axis parallel to that of the rod and that they randomize their orientation within the time taken to make the measurements.

This is obviously a result of great general importance, and in the accompanying article Cone describes measurements that show what the rate of the rotational randomization actually is. Using short flashes to produce an intermediate in the bleaching sequence, and measuring the decay of the ensuing dichroism with perpendicular and parallel polarized light, he has been able to measure, by selection of the appropriate wavelength, the relaxation both of the product of partial bleaching and of the residual unchanged rhodopsin. From the exponential decay rates the rotational relaxation time can be extracted, and this comes to about $20 \mu \mathrm{s}$. Assuming the rhodopsin to be a sphere of about $25 \AA$ radius, it is possible to derive an effective viscosity for the membrane medium in which it is located, and the value so determined is such as would be typical for a light oil.

In the light of these data it seems necessary to reconsider the possibility, which had been tentatively discounted by many workers in the membrane field, that a protein of this size could function in favourable cases as a diffusional ion carrier. 